\title{
Evaluation of Low Cost Fresnel Lens for Portable Concentrated Solar Cell Application
}

\author{
Bo Zhang*, Chaolu Xing and Xiaolin Dai \\ School of Energy Science and Engineering, University of Electronic Science and Technology of \\ China, Chengdu, China \\ * Corresponding Author: bo.zhang@uestc.edu.cn
}

\begin{abstract}
Keywords: Fresnel lens; Solar concentrator; Low cost; Spot size; Energy distribution; Spectrum; Optical efficiency
\end{abstract}

\begin{abstract}
Low cost commercially available Fresnel lens has been investigated as a potential candidate for portable concentrated solar cell application. A numerical model has been developed to simulate the selected Fresnel lens and its spot size, energy distribution, transmission spectrum and optical efficiency were studied systematically. The simulation results indicate that the low cost commercially available lens is suitable for use as a portable solar concentrator if the requirement of optical efficiency is reasonable.
\end{abstract}

\section{Introduction}

The development of solar concentrators has been an important research area since the first use of solar energy in human history. The implementation of solar concentrators indeed offers several advantages, such as improved conversion efficiency, reduced energy cost, and the potential to combine solar thermal and photovoltaics (PV). In PV applications, concentrators are mainly used for the medium- or large-scale power stations, in which cylindrical reflectors or parabolic dish reflectors are usually selected [1-3]. Another type of PV concentration system is the concentrator solar panel. Such panel is consisted of an array of micro-scale concentrators and is directly attached to a solar cell panel. In this case, refractive lens is used instead of reflective collectors, which is quite different from power stations. Besides, there are also other ways for the design of concentrator panels, such as the non-image method using two mirrors and the configuration with an array of small parabolic mirrors $[4,5]$.

Despite of intensive studies on the development of concentration system for power stations, the work on portable concentrated PV is still very limited. In the design of concentrators for such kind of system, specific consideration is needed to achieve a good trade-off between performance and cost. Hence, refraction Fresnel lens is selected for this work since it is much cheaper than reflectors and does not have potential shading problems. In this paper, we will theoretically evaluate the feasibility of using a commercial Fresnel lens for the concentrated PV system. Numerical model will be developed and the spot size, energy distribution, spectrum and optical efficiency of the proposed concentration system will be discussed.

\section{Basic Principles of Fresnel Lens}

A Fresnel lens is similar to a conventional convex lens in both structure and function. Fig. 1 shows a comparison among Plano-convex lens, step lens and Fresnel lens. In a Plano-convex lens, the convex surface refracts sun rays to the focal point while the planar surface does not contribute to the concentration of light. Therefore, the planar part of the convex is intentionally removed in order to reduce material cost and lens thickness and this is actually the step lens. If the left side of a step lens is aligned by translation of each step, a Fresnel lens is formed as shown in Fig. 1(c). Since Fresnel lens is a derivative of Plano-convex lens, it is easy to deduce that it maintains the ability of concentrating light. 


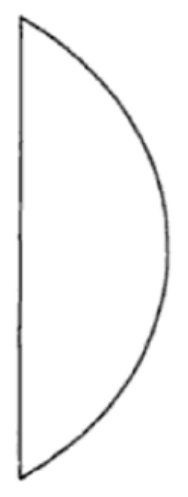

(a)

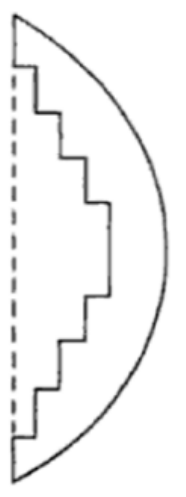

(b)

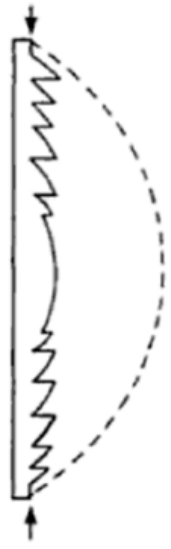

(c)

Fig. 1. (a) Plano-convex lens; (b) Step lens; (c) Fresnel lens.

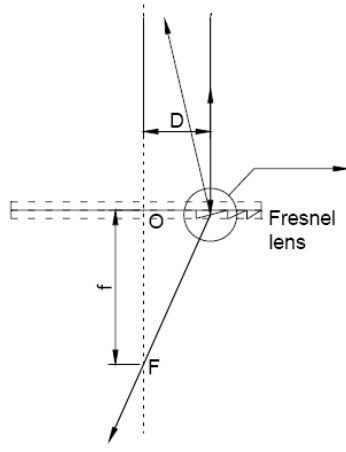

(a)

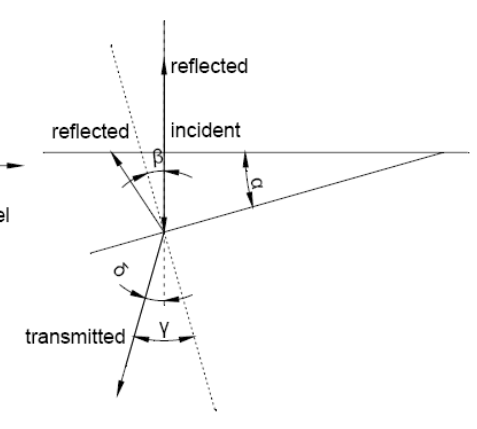

(b)

Fig. 2. (a) Operating principle of the Fresnel lens; (b) A local zoom-in of a facet on the lens.

The operating principle of the Fresnel lens is shown in Fig. 2. In an ideal Fresnel lens, each facet has a different facet angle (the angle " " in Fig. 2(b)). These angles are arranged in such a manner that when parallel rays are incident perpendicular to the lens, all outgoing rays go through the Focal point.

\section{Simulation and Discussions}

The Fresnel lens from Edmund OpticsTM with 8.5 inch X 10.5 inch size and 18 inch focal length was selected for the simulation. Several hypotheses were made as following so that the model can provide better description of the lens.

(1) The lens is perfectly made, for instance, the shape and facet angles of the lens exactly match the design and the grooves are narrow enough as well.

(2)The polymer is ideally uniform and the optical properties agree well with the data in specification.

(3) The tracker is well calibrated and tracks accurately.

(4) The planar side of the lens faces the sun.

Transmittance and Optical Efficiency. The modeling work starts with the reflectance of the lens. Total reflection of Fresnel lens includes reflection from the first surface and the second surface. For the first surface, we can assume a perpendicular incidence for all sun light since sun angular radius is very small [6]. Thus, the S-wave reflectance Rs1 and P-wave reflectance Rp1 are consistent. Thus, the reflectance of the first surface of the lens R1 is:

$$
R_{1}=R_{s 1}=R_{p 1}=\left(\frac{n_{1}-n_{2}}{n_{1}+n_{2}}\right)^{2}
$$

Where $\mathrm{n} 1$ and $\mathrm{n} 2$ are the refractive indexes in the incident and emergent side, respectively. Similarly, the transmittance is same for S-wave and P-wave and the transmittance at the first surface 
is:

$$
T_{1}=T_{s 1}=T_{p 1}=1-R_{1}=\frac{4 n_{1} n_{2}}{\left(n_{1}+n_{2}\right)^{2}}
$$

In order to investigate the light path at the second surface, the exact shape of the grooves is needed, particularly the angle for each facet. According to the data sheet, the focal length of the lens is calibrated at $589 \mathrm{~nm}$ wavelength, which means that all perpendicularly incident light of $589 \mathrm{~nm}$ wavelength should go through the focal point. In Fig. 2, D is the distance from the lens optical axis to an arbitrary perpendicular incident ray and $\mathrm{f}$ is the focal length for $589 \mathrm{~nm}$ wavelength light, which should reach the focal point F. After geometric analysis, the relationships between incident angle , emergence angle , D and $\mathrm{f}$ are revealed as:

$$
\left\{\begin{array}{l}
\frac{\sin \gamma}{\sin \beta}=\frac{n_{2}}{n_{1}} \\
\tan (\gamma-\beta)=\frac{D}{f}
\end{array}\right.
$$

Where $\mathrm{n}_{1}=1, \mathrm{n}_{2}=1.4913$ at wavelength of $589 \mathrm{~nm}, \mathrm{f}=45.72 \mathrm{~cm}$. Above equations are numerically solved and give the relationship between and D as shown in Fig. 3.

Once the value of is obtained, we are able to calculate the reflectance and transmittance for the second surface using following equations for both S-wave (4) and P-wave (5):

$$
\begin{array}{r}
R_{s 2}=\left[\frac{n_{1} \cos \beta-n_{2} \sqrt{1-\left(\frac{n_{1}}{n_{2}} \sin \beta\right)^{2}}}{n_{1} \cos \beta+n_{2} \sqrt{1-\left(\frac{n_{1}}{n_{2}} \sin \beta\right)^{2}}}\right]^{2} \\
R_{p 2}=\left[\frac{n_{2} \cos \beta-n_{1} \sqrt{1-\left(\frac{n_{1}}{n_{2}} \sin \beta\right)^{2}}}{n_{2} \cos \beta+n_{1} \sqrt{1-\left(\frac{n_{1}}{n_{2}} \sin \beta\right)^{2}}}\right]^{2}
\end{array}
$$

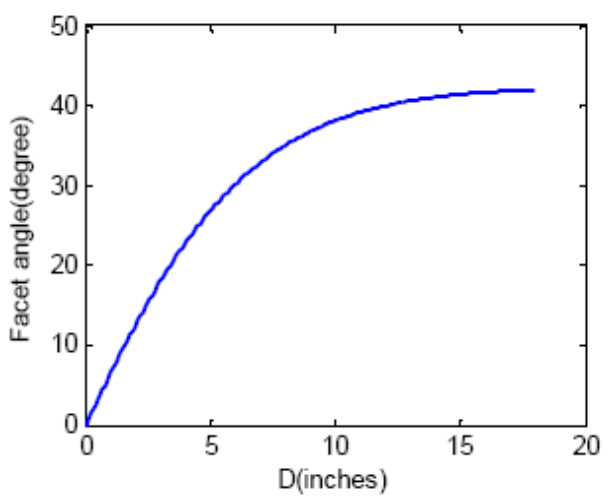

Fig. 3. Facet angle vs. Distance to lens center.

Where $\mathrm{n} 1$ and $\mathrm{n} 2$ are the refractive indexes in incident side and emergent side, respectively. Here, $\mathrm{n} 2=1$ while $\mathrm{n} 1$ is the refractive index of the lens material. Furthermore, natural light contains an equal mix of S- and P-polarisations, so we can get:

$$
R_{2}=\frac{1}{2}\left(R_{s}+R_{p}\right)
$$

then the transmittance of sun light through the whole lens can be written as:

$$
T=T_{1} \times T^{\prime} \times T_{2}=T_{1} \times T^{\prime} \times\left(1-R_{2}\right)
$$

In above expression, $\mathrm{T} 1$ solely depends on the refractive index of the lens; $T$ ' is the transmittance of the lens material obtained for the specification and is only related to the wavelength; and R2 is determined by the refractive index and facet angle. The refractive index of the lens is a function of 
wavelength ( $\mathrm{n}(\quad))$ while the facet angle is a function of distance to lens center ( (D)). Thus, the transmittance $\mathrm{T}$ can be written as $\mathrm{T}(\mathrm{D}, \quad)$ using (7), which is illustrated in Fig. 4.

In order to calculate the overall transmittance of the lens, the integration of $\mathrm{T}(\mathrm{D}, \quad)$ with respect to the active area $\mathrm{C}$ of the lens is performed using (8) and the result is shown in Fig. 5.

$$
T_{\text {total }}(\lambda)=\frac{\iint_{C} T(D, \lambda) \mathrm{d} S}{\iint_{C} \mathrm{~d} S}
$$

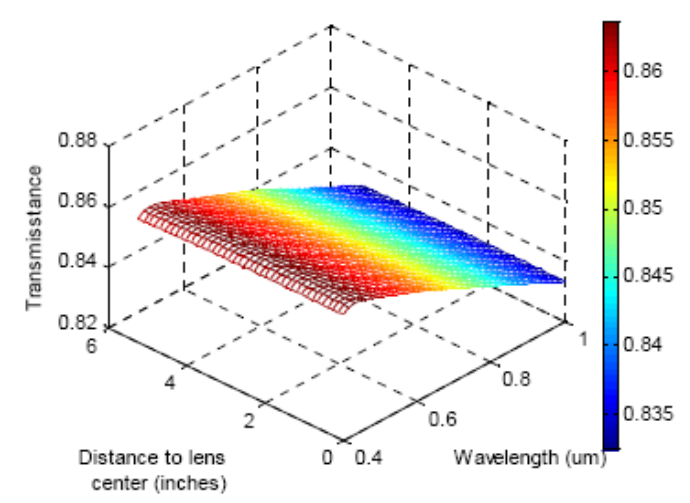

Fig. 4. Local transmittance as a function of wavelength and distance to lens center.

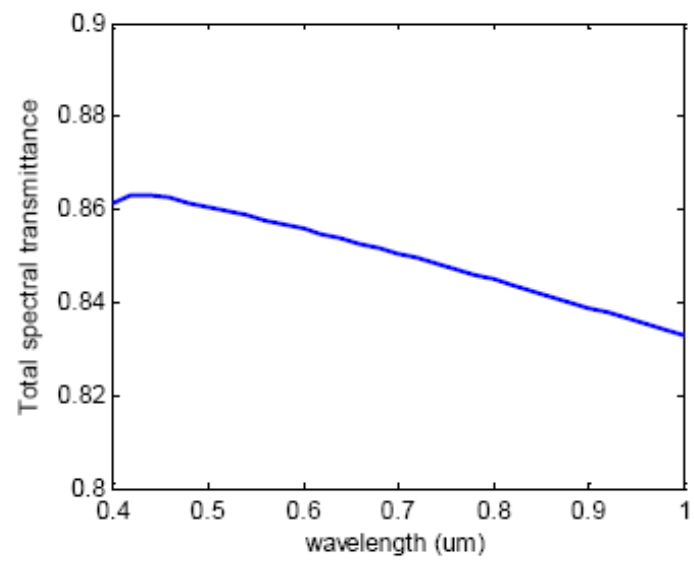

Fig. 5. Total transmittance of the Fresnel lens.

Now the optical efficiency of the lens can be calculated as well, using (9),

$$
\eta_{\text {lens }}=\frac{\int_{400 \mathrm{~nm}}^{1000 \mathrm{~nm}} I(\lambda) \cdot T_{\text {total }}(\lambda) \mathrm{d} \lambda}{\int_{400 \mathrm{~nm}}^{1000 \mathrm{~nm}} I(\lambda) \cdot \mathrm{d} \lambda}
$$

Where $\mathrm{I}(\lambda)$ is the spectral irradiance. For AM 1.5 sun spectrum, the overall lens efficiency is calculated to be about $85.3 \%$ in the spectra range of $400-1000 \mathrm{~nm}$, without considering the spillage on the cell.

Light Spot Size. In real case, not all the sun rays passing through a Fresnel lens reach the same focal point. Several reasons could be responsible for this, such as non-parallel rays, aberration of lens, manufacturing error and Fraunhofer diffraction. In this part of study, the effect of nonparallelism of rays and aberration will be investigated. 


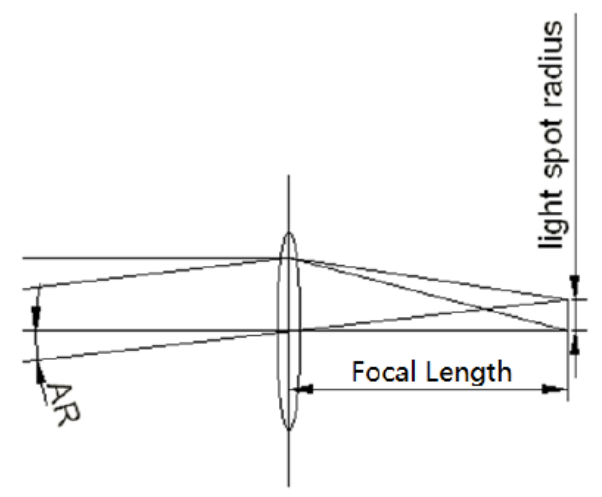

Fig. 6. Illustration of the increase of spot size for non-parallel incident light.

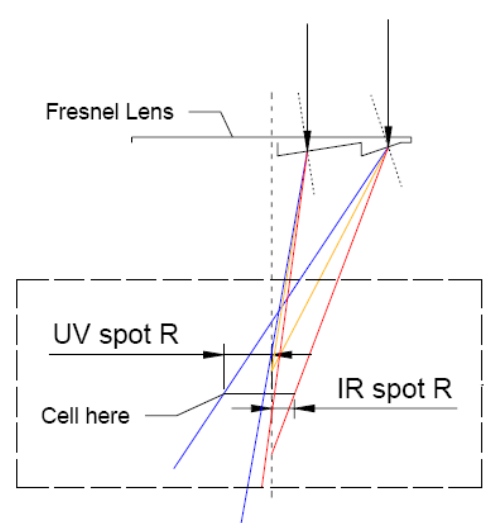

(a)

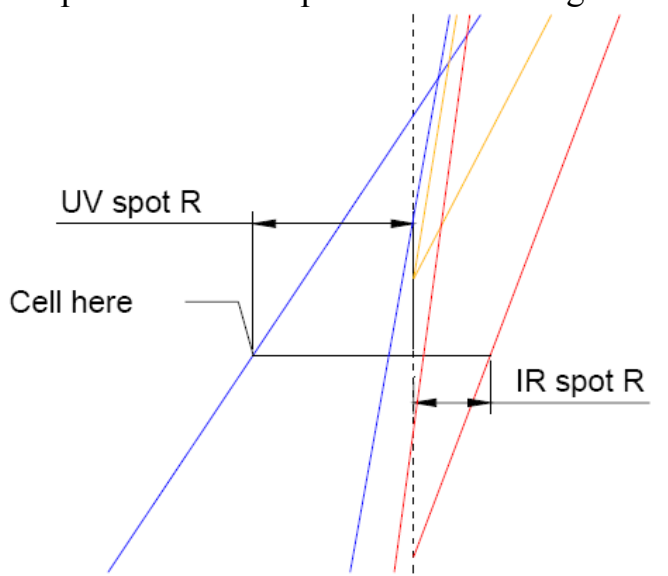

(b)

Fig. 7.(a) Monochromatic aberration combined with chromatic aberration. (b) A local zoom-in of illustration in (a).

Fig. 6 illustrates the contribution of non-parallelism to the increase of light spot radius. Since the incident angle is very small (-960" to 960 "), the comma aberration can be ignored, which is a kind of monochromatic aberration that exists only when the object is not on the optical axis. Therefore, the contribution of non-parallelism is expressed as:

$$
\Delta R_{1}=L \cdot \tan (960 ")
$$

where $\mathrm{L}$ is the distance between the cell and the lens.

Another major contributor to light spot size is chromatic aberration. Its influence on spot size will be first calculated without consideration of non-parallelism effect. Fig. 7 shows the mechanism of chromatic aberration. It is know that the light of different wavelength has different refractive index and it is noticed in the figure that light reaching the edge of lens has the highest aberration. We also find that if the cell is placed between the "bluest" focal point and the "reddest" focal point, the light spot size should be the larger one of the two. If the two are equal, the minimal light spot size is obtained. Up to now, we are able to calculate the light spot size of our lens as both spectra range $(400 \mathrm{~nm}-1110 \mathrm{~nm})$ and groove facet angles are already known. After several steps of geometric and algebraic substitution, the radius of light spot can be written as:

$$
R(D, \lambda, L)=\left|D \times \frac{f(D, \lambda)-L}{f(D, \lambda)}\right|
$$

where $\mathrm{D}$ is the distance from the lens optical axis to an arbitrary perpendicular incident ray and $\mathrm{L}$ is the distance between the cell and the lens, and

$$
f(D, \lambda)=\frac{D}{\tan [\delta(D, \lambda)]}
$$

where $\delta(D, \lambda)=\gamma(D, \lambda)-\beta(D)$ is the deflection angle, and $\gamma(D, \lambda)$ is the emergence angle at the second surface and is expressed as: 


$$
\gamma=\arcsin \left(\frac{n_{2}}{n_{1}} \sin \beta\right)
$$

Equation (14) calculates the overall spot size according to the behavior of sun rays that locate farthest from the optical axis because these rays cause the severest chromatic aberration.

$$
R_{\text {overall }}(L)=\max \left[R\left(D_{\max }, \lambda_{\max }, L\right), R\left(D_{\max }, \lambda_{\min }, L\right)\right](14)
$$

where $D_{\max }=1 / 2\left(\mathrm{a}^{2}+\mathrm{b}^{2}\right)^{1 / 2}, \lambda_{\max }=1110 \mathrm{~nm}, \lambda_{\min }=400 \mathrm{~nm} . \mathrm{R}\left(\mathrm{D}_{\max }, \lambda_{\min }, \mathrm{L}\right)$ and $\mathrm{R}\left(\mathrm{D}_{\max }, \lambda_{\min }, \mathrm{L}\right)$ are the expressions for the UV(400 nm) and IR spot radius, respectively. Obviously, if $L<f\left(D, \lambda_{\min }\right)$, $\mathrm{R}_{\text {overall }}(\mathrm{L})=\mathrm{R}\left(\mathrm{D}_{\max }, \lambda_{\max }, \mathrm{L}\right)>\mathrm{R}\left(\mathrm{f}\left(\mathrm{D}, \lambda_{\min }\right)\right)$; if $\mathrm{L}>\mathrm{f}\left(\mathrm{D}, \lambda_{\max }\right), \mathrm{R}_{\text {overall }}(\mathrm{L})=>\mathrm{R}\left(\mathrm{f}\left(\mathrm{D}, \lambda_{\max }\right)\right)$. Therefore, the value of $L$ that minimizes $R_{\text {overall }}(L)$ must be in the interval $\left[f\left(D, \lambda_{\min }\right), f\left(D, \lambda_{\max }\right)\right]$. In this interval, $\mathrm{R}\left(\mathrm{D}_{\max }, \lambda_{\max }, \mathrm{L}\right)$ is a decreasing function of $\mathrm{L}$ while $\mathrm{R}\left(\mathrm{D}_{\max }, \lambda_{\min }, \mathrm{L}\right)$ is an increasing function of $\mathrm{L}$, which indicates that $\mathrm{R}_{\text {overall }}(\mathrm{L})$ must have its minimal value when these two are equal. Hence, we obtain that the value of $\mathrm{L}$ is $44.63 \mathrm{~cm}$ and the corresponding value of spot size is $0.9085 \mathrm{~cm}$. This is the minimum spot size achievable in theory for perpendicular incident light.

Furthermore, we consider the effects of non-parallelism and aberration together and the radius is expressed as (15).

$$
\begin{aligned}
& R_{\text {combined }}=L \cdot \tan (960 ")+\max \\
& {\left[R\left(D_{\text {max }}, \lambda_{\text {max }}, L\right), R\left(D_{\text {max }}, \lambda_{\text {min }}, L\right)\right.}
\end{aligned}
$$

And we also know that $\tan \left(960^{\prime \prime}\right)<\left|\mathrm{dR}_{\text {overall }}(\mathrm{L}) / \mathrm{dL}\right|$ except at the point $\mathrm{L}=\mathrm{L}_{0}$, which is mathematically not differentiable. So $\mathrm{R}_{\text {combined }}$ is still minimized at $\mathrm{L}=\mathrm{L}_{0}=17.57^{\circ}=44.63 \mathrm{~cm}$ and the minimum radius is $0.4395^{\prime}$ ' $=1.1162 \mathrm{~cm}$. This is the minimum light spot radius achievable by varying the lens-cell distance under sun light, including the effects of aberration and sun angular radius and Fig. 8 shows the influence of aberration and non-parallelism on spot radius. It is noticed that the minimum light spot radius is larger than half the width of usual $1 \mathrm{~cm} \mathrm{x} 1 \mathrm{~cm}$ concentrator cells. Thus, there will be a significant light spillage if no secondary optics is attached. In our case, the most appropriate choice of the secondary optics is the side reflective wall, since it is relatively cheap and easy for installation [7].

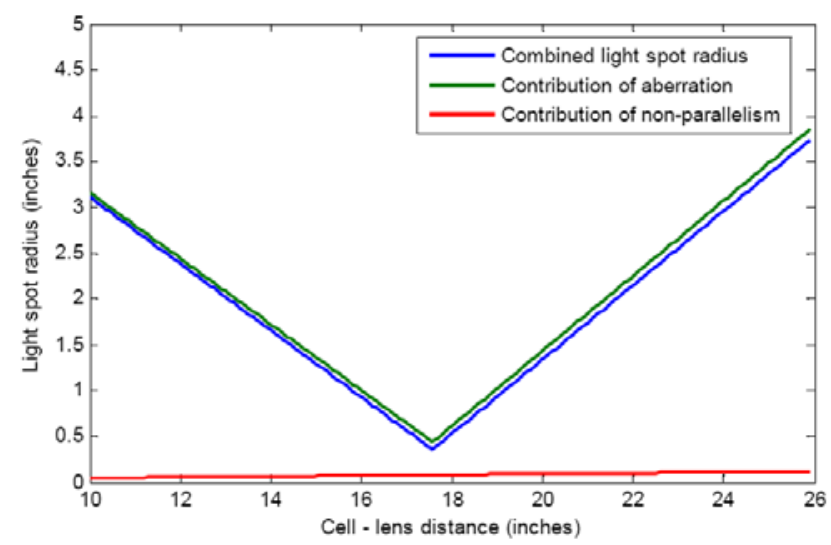

Figure 8. Light spot radius vs. Cell-lens distance.

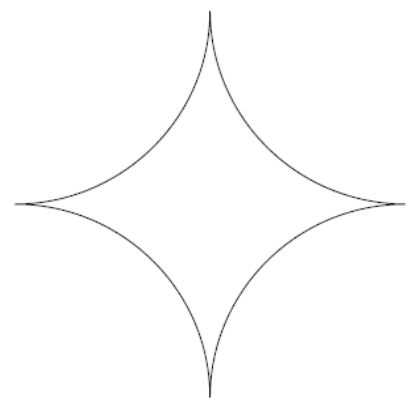

Fig. 9. Shape of the concentrated light spot. 
The shape of light spot is shown in Fig. 9. This kind of shape is mainly caused by the corners of the Fresnel lens. The energy of light would be concentrated near the center of the cell and decreases towards the edges. It is worthy to point out that the light spot size is dependent on the wavelength, which means that there is a distribution of concentrated light for different wavelength, inducing an uneven spectrum distribution.

\section{Conclusions}

Commercial low cost Fresnel lens was evaluated as a concentrator for portable solar cell applications. The optical properties were theoretically investigated using numerical models. The facet angles at different distance from lens center are calculated. The local transmittance of the lens is modeled as a function of wavelength and distance from the lens center. Consequently, the total transmittance as well as the overall optical efficiency is calculated. Furthermore, the influence of non-parallelism and aberration effects on the size and shape of the concentrated light spot is modeled in detail. Our simulation results demonstrate that Fresnel lens is suitable for portable concentrated solar cells with a good trade-off between performance and cost.

\section{Acknowledgements}

This work is supported by the Australia Research Council (ARC) via its Centers of Excellence scheme, and Natural Science for Youth Foundation of China (Contract No. 61106099, No. 51102038)

\section{References}

[1] A. Segal, M. Epstein, and A. Yogev, "Hybrid concentrated photovoltaic and thermal power conversion at different spectral bands", Solar Energy, vol. 76, pp. 59, 2004.

[2] S. A. Kalogirou, "Solar energy engineering: processes and systems", Burlingto San Diego, London: Elsevier/Academic Press, 2009.

[3] T. B. Johansson, and L. Burnham, "Renewable energy: soruces for fuels and electricity", Washington, DC: Island Press, 1993.

[4] P. Benitez, A. Cvetkovic, R. Winston, G. Diaz, L. Reed, J. Cisneros, A. Tovar, A. Ritschel, and J. Wright, "High-concentration mirror-based integrating system for tandem solar cells", IEEE 4th World Conferenceon on Photovoltaic Energy Conversion, pp. 690, 2006.

[5] S. Horne, G. Conley, J. Gordon, D. Fork, P. Meada, E. Schrader, and T. Zimmermann, “A solid 500 sun compound concentrator PV design", IEEE 4th World Conferenceon on Photovoltaic Energy Conversion, pp. 694, 2006.

[6] L. S. Joy, "Gassendi the atomist: advocate of history in an age of science", Cambridge, Cambridge Univ. Press, 2002.

[7] H. P. Garg, "Collection and storage systems: Advances in solar energy technology", vol. 1, Dordrecht [u.a.]: Reidel, 1987. 